\title{
Diskussion
}

Michael Wohlgemuth*

\section{Artikelbearbeitungsgebühren im Spiegel von Forschung und Praxis}

\author{
Eine Replik zu Nina Schönfelder Beitrag: Preistransparenz und -struktur von \\ Artikelbearbeitungsgebühren
}

https://doi.org/10.1515/bfp-2020-2087

Zusammenfassung: Das Spannungsfeld aus Wollen (Abschluss von Transformationsverträgen mit großen Verlagsgruppen, um eine schnelle OA-Transformation in der Breite zu erreichen) und Können (begrenzte Budgets der wissenschaftlichen Bibliotheken) erfordert belastbare Prognosen zur Entwicklung des Publikationsoutputs und der Höhe der Artikelbearbeitungsgebühren. Der Beitrag stellt verschiedene Preissetzungsstrategien von Verlagen vor und diskutiert die Implikationen für statistische Analysen. Abgerundet wird der Beitrag durch den Appell des Autors, die Erwartungen der Bibliotheken an die OATransformation neu zu denken, um eine nachhaltige Finanzierung des Open-Access-Publizierens sicherstellen zu können.

Schlüsselwörter: Open-Access-Transformation; Finanzierung; Artikelbearbeitungsgebühr

\section{Article Processing Charges in the Mirror of Research and Practice}

Abstract: The area of conflict between willingness (conclusion of transformation contracts with large publishing groups in order to achieve rapid OA transformation across the board) and ability (limited budgets of academic libraries) requires reliable forecasts of the development of publication output and the level of article processing fees. The paper presents different pricing strategies of publishers and discusses the implications for statistical analysis. The article is rounded off by the author's appeal to rethink the expectations of libraries with regard to OA transformation in order to ensure sustainable financing of Open Access publishing.

*Kontaktperson: Michael Wohlgemuth, Michael.Wohlgemuth@slub-dresden.de
Keywords: Transition to Open Access; financing; article processing fee

\section{Einleitung}

Ausgehend von Ulrich Herbs Bilanz ${ }^{1}$ lässt sich die OpenAccess-Transformation monetär betrachtet als eine Transformation der Erwartungen zweier zentraler Akteure an Open Access deuten: Die klassischen Verlage haben ihren Widerstand gegen den offenen Zugang zu wissenschaftlichen Publikationen aufgegeben und arbeiten intensiv daran, das Geschäftsmodell Open Access auszuweiten. Und viele wissenschaftliche Bibliotheken verbinden mit Open Access nicht mehr den Anspruch, die Erwerbshaushalte zu entlasten. Stattdessen loten sie stillschweigend mit den Forschungseinrichtungen und Forschungsförderern aus, wie sie unter den gegebenen strukturellen Rahmenbedingungen die „angemessene Bepreisung nach einem einfachen, zukunftsorientierten Berechnungsmodell“ ${ }^{\text {' }}$ in $\mathrm{Zu}-$ kunft tragen können.

Aus Sicht der Verlage liegen die Vorteile klar auf der Hand. Das tradierte subskriptionsbasierte Geschäftsmodell steht durch die digitale Transformation unter starkem Druck. Die alte Paywall (der Leser zahlt) ist im Vergleich zur neuen Paywall (der Autor zahlt) löchrig wie ein Schweizer Käse. Wohl dem Verlag, der die Kosten der Transformation des Geschäftsmodells über festgelegte Vorauszahlungen nationaler Konsortien decken kann. Diese finanzielle Planungssicherheit fehlt den Bibliotheken. Zwar sind in der Übergangszeit der Big Deals die an den Subskriptionsausgaben orientierten Ausgaben für „Publish and Read“ relativ fest fixiert. Die Kosten des Publizie-

1 Herb (2017).

2 Das dritte Verhandlungsziel des Projektes DEAL (https://www.pro jekt-deal.de/aktuelles/), alle Links geprüft am 20.05.2020. 
rens in reinen OA-Zeitschriften müssen laut DEAL-Vertrag aber von den teilnehmenden Einrichtungen zentral bezahlt werden. Noch ist nicht absehbar, wie sich der Publikationsoutput in den DEAL-relevanten Zeitschriften entwickelt. Unbekannt ist zudem, wie schnell und wie viele Zeitschriften von hybrid auf rein OA umgestellt werden, wie sich der Publikationsoutput in diesen Zeitschriften entwickelt und in welcher Höhe die Artikelbearbeitungsgebühren (oder APC) nach der Umstellung anzusetzen sind.

Letztere Frage greift Schönfelder ${ }^{3}$ als Forschungsfrage auf. Ausgehend von der Annahme, dass das Erheben von Artikelbearbeitungsgebühren im Zuge der OA-Transformation weiter an Bedeutung gewinnen wird, analysiert sie Einflussfaktoren, die die Höhe der APC beeinflussen. Das Ergebnis der Analysen zeigt nach Schönfelder, dass die Verlage verschiedene Preissetzungsstrategien für reine OA-Zeitschriften und hybride Zeitschriften verfolgen. Während die Höhe der APC in reinen OA-Zeitschriften stark durch die spezifischen Zitationsimpacts bestimmt wird, sei die Höhe der APC in hybriden Zeitschriften stärker von anderen Einflussfaktoren abhängig (z.B. Verlagsprestige, adressierter Fachbereich).

Der Beitrag Schönfelders zeigt beispielhaft, wie wichtig es ist, Forschung und Praxis zusammenzudenken. Die Aussagekraft der Analyseergebnisse wird durch eine ungeeignete Datenbasis und dadurch bedingte methodische Unschärfen beeinträchtigt, die im Folgenden herausgearbeitet werden sollen.

\section{Artikelbearbeitungsgebühren in der Praxis}

Als im Tagesgeschäft der OA-Finanzierung Tätiger stolpert der Autor in der Einleitung über die Fragestellung von Schönfelders Beitrag: Soll die Höhe der APC nach einem umfassenden Journal-Flipping abgeschätzt werden ${ }^{4}$ oder soll analysiert werden, welche Faktoren „die Höhe von tatsächlich gezahlten Artikelbearbeitungsgebühren (in Gegensatz zu Listenpreisen) beeinfluss[en]“"5?

Schönfelder grenzt an dieser Stelle die tatsächlich bezahlten APC aus dem OpenAPC-Datensatz von den Listenpreisen der Verlage ab. Leider lässt sie offen, worin das Wesen dieses Unterschiedes besteht. Die Auswahl der vor-

3 Schönfelder (2020).

4 Diese Frage bezieht sich auf die Listenpreise.

5 Schönfelder (2020) 22. gestellten potentiellen Einflussfaktoren lässt erkennen, dass sich Schönfelder dieser Unterschiede nicht bewusst ist. Anders kann sich der Autor nicht erklären, dass in dieser Auflistung der zumindest für Deutschland zentrale Faktor „Individuelle Rabattverhandlung“ fehlt. Die gestellte Forschungsfrage erfordert es jedoch, sich systematischer mit diesen Unterschieden auseinanderzusetzen.

Die Listenpreise von reinen OA-Journalen können innerhalb eines Verlages sehr stark variieren. ${ }^{6}$ Die Preisinformationen liegen dann statistisch ausgedrückt als metrische Merkmale vor. Dieses Skalenniveau erlaubt es, Aussagen zur Häufigkeit, der Rangfolge und dem Abstand der Preise untereinander zu treffen. In anderen Verlagen lassen sich eine Handvoll Preiskategorien beobachten, denen die Journale zugeordnet sind. ${ }^{7}$ Derartige Preise werden in den Wirtschaftswissenschaften als Staffelpreise oder Qualitätsstaffeln bezeichnet. Auch wenn Preise grundsätzlich als metrische Merkmale bezeichnet werden, sprechen einige Argumente dafür, diese Staffelpreise als pseudometrische Merkmale zu bezeichnen, da diese Preise im Rahmen der Preisbildung den ordinalen Merkmalsausprägungen von ausgewählten Einflussfaktoren zugewiesen wurden und der Gliedabstand der Preise in der Reihe bzw. Staffel demzufolge nur eine bedingte Aussagekraft hat (besser oder schlechter als X).

Der Verlag Frontiers eignet sich hervorragend, um diesen Sachverhalt aufzuzeigen. ${ }^{8}$ Tabelle 1 zeigt, dass Frontiers seine Journale in fünf Kategorien und jede in jeweils vier Artikeltypen einteilt, die spezifisch bepreist werden.

Tab. 1: Preiskategorien des Verlages Frontiers

\begin{tabular}{llrrrr}
\hline $\begin{array}{l}\text { Cate- } \\
\text { gory }\end{array}$ & Description & $\begin{array}{r}\text { Article } \\
\text { type A }\end{array}$ & $\begin{array}{r}\text { Article } \\
\text { type B }\end{array}$ & $\begin{array}{r}\text { Article } \\
\text { type C }\end{array}$ & $\begin{array}{r}\text { Article } \\
\text { type D }\end{array}$ \\
\hline 1 & $\begin{array}{l}\text { Newly launched } \\
\text { journals and/or those } \\
\text { in fields that have } \\
\text { lower research budgets }\end{array}$ & $\$ 950$ & $\$ 700$ & $\$ 450$ & $\$ 0$ \\
& & & & & \\
\hline 2 & $\begin{array}{l}\text { Recently launched } \\
\text { journals and/or those } \\
\text { in fields with moderate } \\
\text { research budgets }\end{array}$ & $\$ 1,150$ & $\$ 700$ & $\$ 450$ & $\$ 0$ \\
& & & & \\
\hline
\end{tabular}

6 Vgl. dazu die DEAL-Preisinformationen von Wiley: https://keeper. mpdl.mpg.de/f/1578cfa1ea894d50970f/?dl=1.

7 Vgl. dazu die Preisinformationen von De Gruyter: https://www.deg ruyter.com/page/2046?language=en oder MDPI: https://www.mdpi.c om/apc.

8 Vgl. dazu die Preisinformationen von Frontiers: : https://www.fron tiersin.org/about/fee-policy. 
Tab. 1: (fortgesetzt)

\begin{tabular}{llrrrr}
\hline $\begin{array}{l}\text { Cate- } \\
\text { gory }\end{array}$ & Description & $\begin{array}{r}\text { Article } \\
\text { type A }\end{array}$ & $\begin{array}{c}\text { Article } \\
\text { type B }\end{array}$ & $\begin{array}{r}\text { Article } \\
\text { type C }\end{array}$ & $\begin{array}{r}\text { Article } \\
\text { type D }\end{array}$ \\
\hline 3 & $\begin{array}{l}\text { Journals that are well- } \\
\text { established and in } \\
\text { fields that support } \\
\text { Open Access }\end{array}$ & $\$ 1,900$ & $\$ 875$ & $\$ 450$ & $\$ 0$ \\
\hline 4 & $\begin{array}{l}\text { Mature and impactful } \\
\text { journals that are well- } \\
\text { established and in } \\
\text { fields that support }\end{array}$ & $\$ 2,490$ & $\$ 1,150$ & $\$ 450$ & $\$ 0$ \\
& Open Access & & & & \\
\hline 5 & $\begin{array}{l}\text { Sustainable and } \\
\text { impactful journals in } \\
\text { fields that support } \\
\text { Open Access }\end{array}$ & $\$ 2,950$ & $\$ 1,850$ & $\$ 450$ & $\$ 0$ \\
& & & & \\
\hline
\end{tabular}

Die Spalte Description lässt erkennen, dass sich Frontiers in der Bepreisung der Kategorien stark an den klassischen „Qualitätsindikatoren“ Sichtbarkeit und Impact des wissenschaftlichen Betriebes oder aber der Zahlungskraft der adressierten Fachbereiche (Kategorie 2) orientiert. Die Preise der fünf Kategorien liegen sichtlich an der erzielbaren Obergrenze. Das Beispiel Frontiers zeigt, dass die Höhe des Zitationsimpacts nicht direkt die Höhe der Artikelbearbeitungsgebühren beeinflusst. Preisrelevant ist hier, ob eine Zeitschrift in den Journal Citation Reports indexiert ist und somit einen JIF aufweist, oder nicht. Auch die Listenpreise des Verlages Copernicus entsprechen Qualitätsstaffeln. ${ }^{9}$ Im Gegensatz zu Frontiers erfolgt hier die Bepreisung aber nicht artikel-, sondern seitenbasiert. Zusätzlich gibt es Rabatte für Mitglieder in Fachgesellschaften. Dieser Verlag orientiert sich in der Preisbildung jedoch nicht an den oben beschriebenen „Qualitätskriterien“, sondern an den Kosten der Produktion ${ }^{10}$ sowie dem eventuellen Wunsch der Journaleigner, über das Journal Einnahmen zu generieren.

Die realen APC aus dem OpenAPC-Datensatz unterscheiden sich nicht nur von Verlag zu Verlag und Zeitschrift zu Zeitschrift. Diese realen APC können auch innerhalb einer Zeitschrift stark variieren. Diese Differenzen deuten in der Regel auf Preisdifferenzierungen, ${ }^{11}$ variieren-

9 https://publications.copernicus.org/for_authors/article_proces sing_charges.html.

10 https://publications.copernicus.org/apc_information.html.

11 Diese Preisdifferenzierungen sind eine Methode der Preispolitik und dienen langfristig dem unternehmerischen Erfolg der Verlage. Um neue Märkte zu erschließen (Einführung neuer Zeitschriften bzw. Eroberung von Marktanteilen) oder aber Autoren als Gutachter zu gewinnen bzw. zu halten, kann es für Verlage sinnvoll sein, die APC de Abrechnungsmodelle wie eine Abrechnung nach Seitenanzahlen oder Dokumententypen, umrechnungsbedingte Währungsschwankungen, eine Befreiung von der Umsatzsteuer oder das Jahr der Abrechnung hin.

Im Gegensatz zu den Listenpreisen erscheinen die realen APC des OpenAPC-Datensatzes auf den ersten Blick metrisch skaliert. Auch wenn die realen APC in OpenAPC teilweise deutlich von den Listenpreisen abweichen und stark variieren, sind sie in Abhängigkeit der Preissetzungsstrategien der Verlage aber weiterhin in Teilen als pseudometrische bzw. ordinalskalierte Merkmale zu betrachten.

Der große Wert des OpenAPC-Datensatzes liegt darin, Preisdifferenzen auf Artikelebene zwischen Zeitschriften und innerhalb von Zeitschriften abzubilden. Eine Kenntnis dieser Differenzen erlaubt es, den für die OA-Finanzierung Zuständigen in einen Informationsaustausch mit anderen Einrichtungen einzutreten, um z.B. von deren Erfahrungen in Rabattverhandlungen mit Verlagen zu profitieren. Aus Sicht der Praxis ist jede einzelne erfolgreiche Verhandlung ein Beitrag zur langfristigen Finanzierung des OpenAccess-Publizierens. Die Preisdifferenzen ${ }^{12}$ zwischen Artikeln einer Zeitschrift erschweren es aber gleichzeitig, belastbare Aussagen zu Einflussfaktoren auf die APC einer Zeitschrift treffen zu können. Schönfelder versucht, die benannten Einflüsse auszuschließen und die Regression auf Basis einer repräsentativen Stichprobe zu analysieren. Da die britischen Preisinformationen im OpenAPC-Datensatz nach Schönfelder nicht durch „Einschränkungen hinsichtlich der Förderfähigkeit bzw. der Kostenübernahme“"13 verzerrt werden, basiert ihre Regressionsanalyse auf diesen Teildatensatz der Jahre 2014-2016. Die britischen Daten dieser Jahre stammen laut OpenAPC-Darstellung ${ }^{14}$ aus der Jisc collection oder vom Wellcome Trust. Dieses Sample kann aus Sicht des Autors aber nicht als repräsentative Stichprobe einer Grundgesamtheit bezeichnet werden, da über die Komposition der Daten keine Informationen vorliegen ${ }^{15}$ und in Kenntnis der Daten davon auszugehen ist, dass nur ein Teil des Publikationsoutputs der britischen Universitäten über die Jisc collection erfasst wird.

zu rabattieren. Der Autor betrachtet auch Rabatterfolge, die durch den Verweis auf die DFG-Kostenobergrenze erzielt wurden, als Bestandteil der Preisdifferenzierung.

12 Im OpenAPC-Datensatz sichtbar über die ausgewiesene Standardabweichung ausgewiesen.

13 Schönfelder (2020) 27.

14 https://github.com/OpenAPC/openapc-de/tree/master/data/jis c_collections/2014-2016\#2-preprocessed.

15 Laut Eigendarstellung verhandelt Jisc mit kommerziellen Verlagen Open-Access-Verträge (https://www.jisc-collections.ac.uk/Abo ut-JISC-Collections/Supporting-transition-to-Open-Access/). 


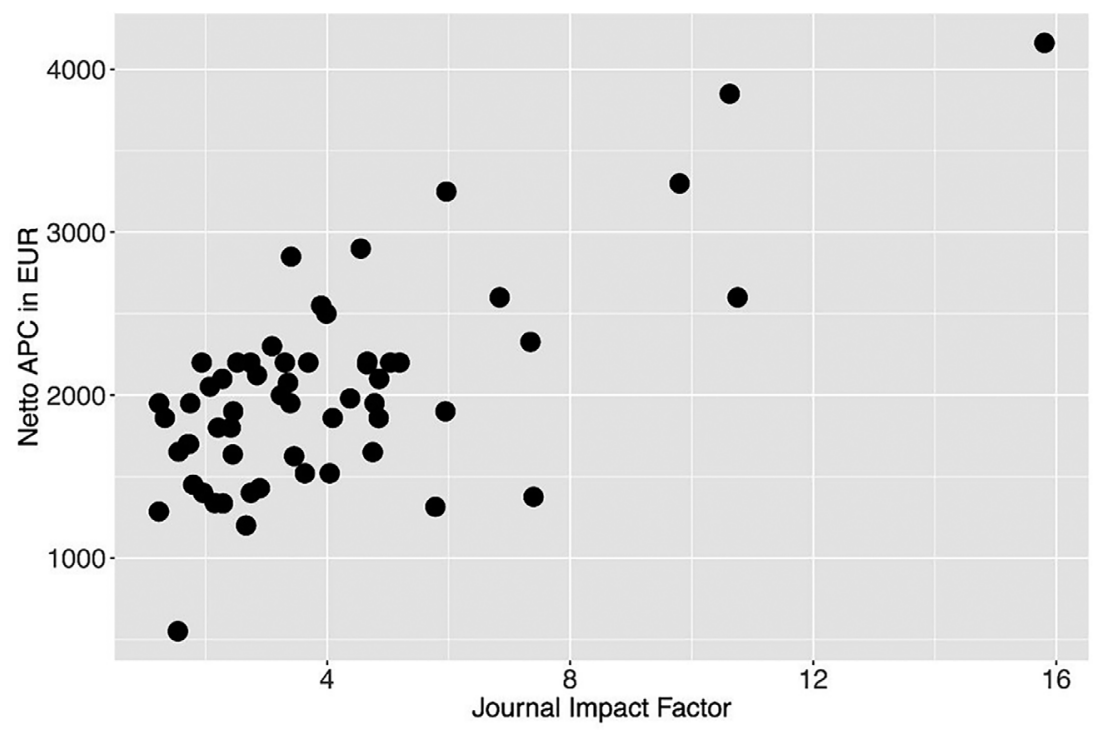

Abb. 1: Streudiagramm aktuelle Listenpreise (Wiley) und JIF

\section{Artikelbearbeitungsgebühren im Modell}

Der vorangestellte Überblick zur praktischen Natur der Artikelbearbeitungsgebühren zeigt einerseits, dass die von Schönfelder gestellte Forschungsfrage nur auf Basis der Listenpreise beantwortet werden kann. Der für manchen Leser trockene Exkurs zu den Skalenniveaus der Preisinformationen erscheint dem Autor notwendig, da die Anwendung statistischer Verfahren zur Bestimmung von potentiellen Einflussfaktoren eine präzise Bestimmung des Skalenniveaus der untersuchten Merkmale (hier APC) voraussetzt. Schönfelder untersucht in Abschnitt 2 die Korrelation zwischen Zitationsimpact und den realen APC und stellt diesen Zusammenhang als hoch signifikant dar. Es fehlen Angaben zum Korrelationskoeffizienten und dem Signifikanzniveau, die helfen, diese Aussage einzuordnen. Die von Schönfelder wahrscheinlich verwendete Korrelationsanalyse nach Pearson erfordert zwei metrisch skalierte Merkmale, die dann zueinander ins Verhältnis gesetzt werden. Dieses Analyseverfahren erlaubt es jedoch nicht, einen Zusammenhang von pseudometrischen Preisinformationen und metrisch skalierten Merkmalen wie dem Zitationsimpact aufzuzeigen. ${ }^{16}$ Ein Einfluss des Zitations-

16 Der Autor hat dieses Analyseverfahren selbst fälschlicherweise in einer Analyse eingesetzt, die den Zusammenhang zwischen Listenpreisen und JIF untersuchte und im Rahmen der Grazer OA-Tage 2018 vorgestellt wurde (Wohlgemuth, Michael; Adam, Michaele; Musiat, Jutta (2018, October). Der Journal Impact Factor auf dem Prüfstand: impacts auf die Höhe der APC lässt sich mit diesem Verfahren aber nur dann aufzeigen, wenn die Analyse auf metrisch skalierten Preisinformationen aufbaut.

Abbildung 1 stellt den aktuellen Journal Impact Factor (JIF) von Clarivate ${ }^{17}$ und die entsprechenden Listenpreise von Gold-OA-Journalen des Verlages Wiley in ein Verhältnis. Als Basis dieser Analyse wurden alle Daten des Science Citation Index und des Social Science Citation Index über die ISSN mit den Daten der Keeper-Liste von Wiley ${ }^{18}$ verbunden. Der Datensatz umfasst Preis- und Impactinformationen von insgesamt 59 Journalen, von denen 9 im Rahmen des DEAL-Vertrages auf ein reines OA-Geschäftsmodell umgestellt (geflippt) wurden.

Jeder Punkt im Streudiagramm entspricht einem Journal. Der lineare positive Zusammenhang zwischen beiden metrischen Variablen ist sichtbar und wird im Rahmen einer Korrelationsanalyse (nach Pearson) durch den Korrelationskoeffizienten von 0,7 bestätigt. Über diesen beschriebenen positiven Zusammenhang hinaus erlaubt das Verfahren keine kausalen Aussagen. Der beobachtete $\mathrm{Zu}$ sammenhang kann also auch zufällig sein.

Grenzen und Potentiale in der Qualitätsbewertung von OA-Zeitschriften. In: Zenodo. http://doi.org/10.5281/zenodo.1441258).

17 Die von Schönfelder (2020) erwähnte Schwäche des JIFs in Bezug auf die Vergleichbarkeit dieses Indikators über Fachbereiche hinweg ist für diese Analyse bedeutungslos. Festzuhalten bleibt, dass der JIF von den meisten Verlagen und Autoren weiterhin als zentraler Impactindikator wahrgenommen wird.

18 https://keeper.mpdl.mpg.de/f/1578cfa1ea894d50970f/?dl=1. 


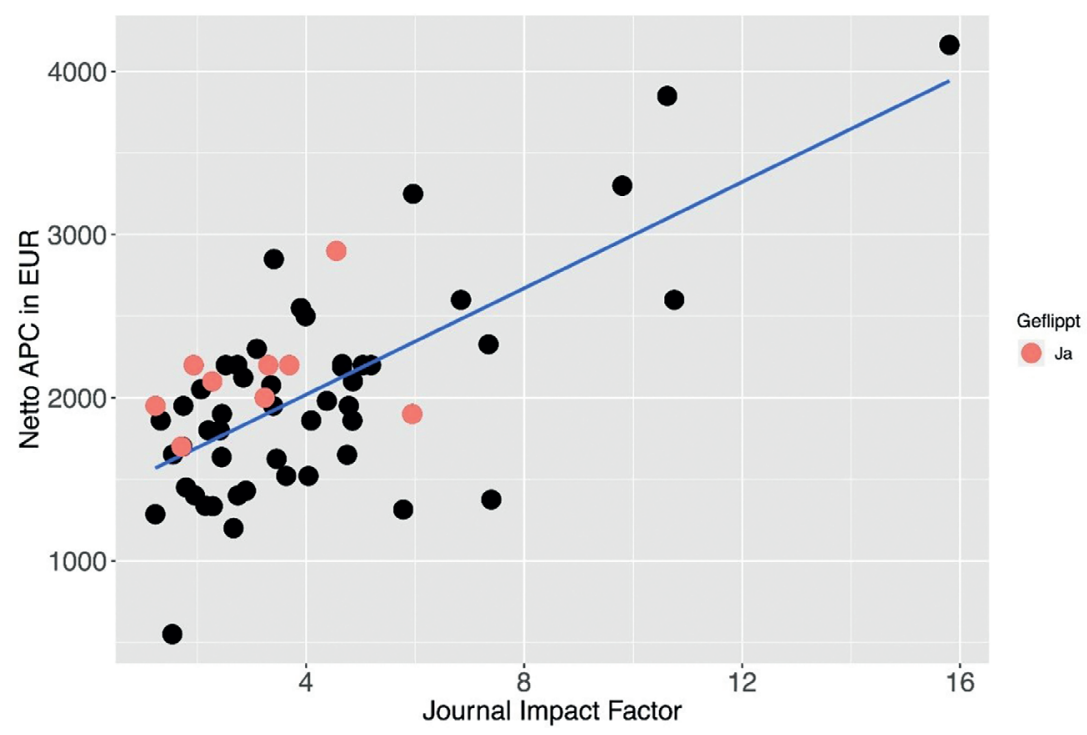

Abb. 2: Streudiagramm aktuelle Listenpreise (Wiley) und JIF mit Regressionsgerade

Auf Basis einer Regressionsanalyse ist es in einem nächsten Schritt möglich, die Entwicklung einer sogenannten abhängigen Variable durch eine unabhängige oder erklärende Variable zu prognostizieren. Im vorliegenden Beispiel soll der Listenpreis (abhängige Variable) durch den JIF (unabhängige Variable) erklärt werden. Die Regressionsanalyse ist somit geeignet, Hinweise auf die Preissetzungsstrategie des Verlages Wiley zu geben. Abbildung 2 entspricht im Aufbau weitgehend Abbildung 1. Ergänzend wurde die Regressionsgerade eingezeichnet. Das Bestimmtheitsmaß von 0,48 erlaubt die Aussage, dass fast $50 \%$ der Streuung durch das sogenannte lineare Modell erklärt wird. Das Bestimmtheitsmaß ist dabei ein reines Zusammenhangsmaß und ebenfalls nicht geeignet, kausale Zusammenhänge zwischen Listenpreis und JIF nachzuweisen.

In Abbildung 2 sind die Journale rot hervorgehoben, die im Rahmen des DEAL-Vertrages geflippt wurden.

Mit Ausnahme der Zeitschrift Ecography liegen alle geflippten Journale über der Regressionsgeraden. Das bedeutet, dass fast alle geflippten Journale höhere APC aufweisen, als der lineare Zusammenhang zwischen Zitationsimpact und Listenpreisen es hätte vermuten lassen. Tabelle 2 stellt die APC der geflippten Journale nach Listenpreisen den APC gegenüber, die sich auf Basis des Regressionsmodells berechnen lassen.
Tab. 2: APC in Flipping Journals von Wiley

\begin{tabular}{lccr}
\hline Zeitschrift & JIF & Listenpreis & $\begin{array}{r}\text { APC nach } \\
\text { Regressions- } \\
\text { modell }\end{array}$ \\
\hline ECOGRAPHY & 5,946 & $1.900 €$ & $2.336 €$ \\
\hline INTERNATIONAL JOURNAL & 2,276 & $2.100 €$ & $1.738 €$ \\
OF METHODS IN & & & \\
PSYCHIATRIC RESEARCH & & & \\
\hline HUMAN BRAIN MAPPING & 4,554 & $2.900 €$ & $2.109 €$ \\
\hline ANNALS OF NONINVA- & 1,235 & $1.950 €$ & $1.569 €$ \\
$\begin{array}{l}\text { SIVE ELECTROCARDIO- } \\
\text { LOGY }\end{array}$ & & & \\
\hline METEOROLOGICAL & 1,711 & $1.700 €$ & $1.646 €$ \\
APPLICATIONS & & & \\
\hline $\begin{array}{l}\text { ENGINEERING IN LIFE } \\
\text { SCIENCES }\end{array}$ & 1,936 & $2.200 €$ & $1.683 €$ \\
\hline $\begin{array}{l}\text { MATERNAL \& CHILD } \\
\text { NUTRITION }\end{array}$ & 3,305 & $2.200 €$ & $1.906 €$ \\
\hline SPACE WEATHER & 3,691 & $2.200 €$ & $1.969 €$ \\
\hline JOURNAL OF FLOOD RISK & 3,240 & $2.000 €$ & $1.895 €$ \\
MANAGEMENT & & & \\
\hline & & & \\
\hline
\end{tabular}

Da auch die geflippten Journale einen positiven linearen Zusammenhang zwischen Listenpreis und JIF erkennen lassen, lässt sich vermuten, dass Wiley das Flipping nutzt, um die Artikelbearbeitungsgebühren der neuen OA-Zeitschriften auf ein höheres Preisniveau zu heben. 


\section{Zusammenfassung}

Das Spannungsfeld aus Wollen (Abschluss von Transformationsverträgen mit großen Verlagsgruppen, um eine schnelle OA-Transformation in der Breite zu erreichen) und Können (begrenzte Budgets der wissenschaftlichen Bibliotheken) erfordert eine genaue Kenntnis der zukünftigen Belastungen der Erwerbshaushalte. Diese Belastungen setzen sich aus zwei unbekannten Faktoren zusammen: Der Zahl der Publikationen sowie der Höhe des Preises (Artikelbearbeitungsgebühren). Schönfelder wirft in ihrem Beitrag die Frage nach der zukünftigen Höhe der APC auf und stellt auf Basis einfacher statistischer Verfahren (Korrelationsanalyse, Regressionsanalyse) heraus, dass die Höhe der APC in reinen OA-Journalen wesentlich durch den Zitationsimpact beeinflusst wird. Ein solcher Debattenbeitrag ist grundsätzlich wertvoll und das Ergebnis scheinbar plausibel. Das Erfahrungswissen aus der OA-Finanzierungspraxis hilft jedoch zu erkennen, dass die Aussagekraft der Analyseergebnisse durch eine für diese Forschungsfrage ungeeignete Datenbasis und methodische Unschärfen beeinträchtigt wird. Der von Schönfelder als Kausalität interpretierte positive Zusammenhang zwischen Artikelbearbeitungsgebühren und dem Zitationsimpact erklärt lediglich 50\% der Varianz in der Höhe von APC in WileyZeitschriften. Die gesonderte Betrachtung der geflippten Zeitschriften lässt darüber hinaus die Annahme zu, dass der Verlag das Flipping nutzt, um ein insgesamt höheres Preisniveau einzuführen. Andere Verlage setzen in ihrer Preissetzungsstrategie wiederum auf Staffelpreise, deren Höhe sich an Kriterien orientiert, die leider immer noch zur Leistungsmessung in der Wissenschaft eingesetzt werden.

Die praktische Relevanz solcher Erkenntnisse ist hoch, da es erst die Kenntnis der Preissetzungsstrategien des Verhandlungspartners ermöglicht, Rahmenverträge auszuhandeln, an denen möglichst viele Bibliotheken teilnehmen können, ohne ihre Angebote an anderer Stelle einkürzen zu müssen. Die aufgezeigten Probleme an der Datenbasis lassen es als notwendig erscheinen, eine möglichst flexible Datenbank aufzubauen (oder OpenAPC entsprechend zu erweitern), auf der die vorhandenen Preis- informationen der Verlage abgebildet werden können. Eine solche Datenbasis erlaubt es, den Einsatz statistischer Verfahren auf die Preissetzungspolitik eines jeden einzelnen Verlages abzustimmen und immer wieder neu anzupassen. Von Nutzen ist es, dabei das Erfahrungswissen der Erwerbs- und OA-Teams über die Grenzen der eigenen Einrichtung hinaus einzubinden.

Der Abschluss der DEAL-Verträge ändert das Zusammenspiel zwischen Verlagen und Bibliotheken im Rahmen der OA-Transformation grundsätzlich. Über die für viele Einrichtungen weiterhin notwendige (und erfolgreiche) Verhandlung von Rabatten und Rahmenverträgen hinaus sind die Bibliotheken aufgefordert, die Erwartungen an die OA-Transformation neu auszuformulieren. Daraus lassen sich dann Zielsetzungen und Handlungspfade ableiten, die - flankierende Rahmenbedingungen der Forschungsförderer und des Wissenschaftsbetriebes vorausgesetzt Einfluss auf Höhe und Durchlässigkeit der neuen Paywalls der Verlagspartner haben werden.

\section{Literaturverzeichnis}

Herb, Ulrich (2017): Open Access zwischen Revolution und Goldesel. In: Information - Wissenschaft \& Praxis, 68 (1). DOI: https://doi. org/10.1515/iwp-2017-0004

Schönfelder, Nina (2020): Preistransparenz und -struktur von Artikelbearbeitungsgebühren. In: BIBLIOTHEK - Forschung und Praxis, 44 (1). DOI: https://doi.org/10.1515/bfp-2019-2079

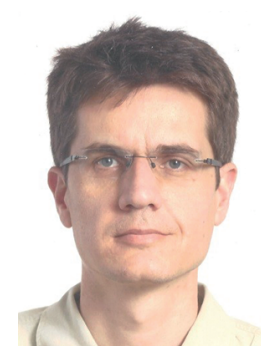

Michael Wohlgemuth

Stabsstelle Publikationsfonds und OpenAccess-Publizieren

Sächsische Landesbibliothek - Staats- und Universitätsbibliothek Dresden

Abteilung Bestandsentwicklung und

Metadaten

D-01054 Dresden

Michael.Wohlgemuth@slub-dresden.de https://orcid.org/0000-0002-7280-5284 